Pediat. Res. 3: 538-544 (1969)

Adolescence follicle stimulating

hormone

gonadotropins radioimmunoassay

serum luteinizing

hormone

\title{
Determination of Serum Luteinizing Hormone (SLH) by Radioimmunoassay in Disorders of Adolescent Sexual Development
}

\author{
Harvey J. Guyda, Ann J.Johanson, Glaude J. Migeon, and Robert M.Blizzard ${ }^{[28]}$ \\ The Harriet Lane Service of the Children's Medical and Surgical Center \\ and The Johns Hopkins University School of Medicine, Baltimore, Maryland, USA
}

Extract

Serum luteinizing hormone (SLH) values in children with various clinical disorders of sexual development are reported and compared with serum levels in normal people at various ages. Values were determined by a double antibody radioimmunoassay method.

In 51 patients aged 2-38 years with gonadal dysgenesis, 131 serial determinations revealed a significant increase in mean SLH at a prepubertal age of 2-9 years, as compared with the mean SLH in control patients in the same age range (fig. 1). Beyond 9 years of age, most values were greater than the normal levels for these ages. In $50 \%$ of the patients 16 years of age and older, cyclic estrogen therapy $(1.25 \mathrm{mg} / 24 \mathrm{~h}$ ) did not suppress the markedly elevated SLH values.

In isosexual precocity, the mean of 48 SLH determinations in 34 females $\left(2^{3} / 12^{-12^{10} / 12}\right.$ years of age) was significantly increased for age in both menstruating and nonmenstruating patients (fig. 2). The mean SLH for 13 values obtained from nine patients receiving Depo-provera (medroxyprogesterone) was significantly higher than that of control patients of comparable age. SLH values for all patients with idiopathic sexual precocity were in the normal range for stage of sexual development, irrespective of age.

SLH in nine patients with premature pubarche and in eight with premature thelarche were in the normal range for age (fig. 3). In 52 boys with constitutional delay of growth and adolescence, the mean of 95 serial determinations was significantly lower than the mean SLH for comparable ages (fig.4). The values increased as sexual development advanced. SLH values for six patients with adolescent gynecomastia were in the normal range for age.

Measurement of SLH by radioimmunoassay correlated well with clinical status. Determination of SLH may help evaluate patients with clinical disorders of adolescent sexual development.

\section{Speculation}

The measurement of gonadotropins using the radioimmunoassay technique can provide valuable information for base-line studies. Such studies are preparatory and necessary to investigate and elucidate the interrelation of the cerebral cortex, hypothalamus, pituitary, adrenals, and gonads. It is anticipated that centers of inhibition and secretion, under both hormonal and emotional control, will be found and that the physiology and pathophysiology of normal and abnormal adolescent development will be better understood. 


\section{Introduction}

Radioimmunoassay techniques have previously been utilized to measure serum gonadotropins, luteinizing hormone and follicle stimulating hormone ( $\mathrm{LH}$ and FSH), in normal male and female children and adolescents $[8,16]$. A progressive increase in serum levels of both $\mathrm{LH}$ and FSH occurs with increasing age and normal sexual development. Such data can be used for comparison with serum levels of gonadotropins found in children with various disorders of adolescent sexual development. As a result, the physiology and pathophysiology of both normal and abnormal adolescence may be better understood. In this report, SLH values in children with various clinical disorders of sexual development are reported and compared with serum levels in normal people at various ages.

\section{Subjects}

\section{Patient Material}

The patients with abnormalities of sexual development were seen in the Pediatric Endocrine Clinic of the Johns Hopkins Hospital from 1964 to the present. Sera were obtained from all patients seen in this clinic and stored at $-20^{\circ}$. Selection of patients was made on the basis of available sera from this collection. Normal male values were obtained from 100 boys, 5-18 years of age, who were between the 25 th and 75 th percentiles for growth; normal female values were obtained from 148 girls, 2-20 years of age [8]

Gonadal dysgenesis. One hundred and thirty-one determinations of SLH were performed on serially obtained venous blood samples from 51 patients with gonadal dysgenesis, 2-38 years of age. The karyotypes of 14 were $45 / \mathrm{XO}$. Eleven had mosaicism, 3 were $\mathrm{X}$-iso-X, 2 presumed mosaics were 3 and $8 \%$ sexchromatin positive on buccal smear, respectively, and 1 had pure gonadal dysgenesis with a karyotype of $46 / X X$. The cells from 20 patients had not been karyotyped and were sex-chromatin negative.

By age 13, most of these patients were receiving Halotestin (fluoxymesterone) to promote growth [7]. Since no effect was observed on the level of mean SLH from this drug at dosages used, this group is not further delineated. Nineteen determinations were obtained while 15 of the patients were taking estrogen therapy for periods up to 20 years.

Sexual precocity, premature pubarche, and premature thelarche. Forty-eight determinations of SLH were made on 34 females $\left(2^{3} / 12^{-}-12^{10} / 12\right.$ years of age $)$ with isosexual precocity; sexual precocity was defined as the onset of sexual development prior to age 8 years, or the onset of menarche prior to age 10 . In addition to sexual pre- cocity, all patients, except for two, had advanced physical and skeletal development. The exceptions included one patient with McGune-Albright's syndrome who had normal height age (HA) and bone age (BA) and one with hypothyroidism who had delayed HA and BA. Twenty-nine patients had idiopathic sexual precocity, 3 had organic central nervous system (CNS) disease, 1 had McCune-Albright's syndrome, and 1 had hypothyroidism resulting from chronic lymphocytic thyroiditis.

SLH determinations were also made on nine patients, 14 months-8 years of age, with premature pubarche (precocious axillary and pubic hair only) and on eight patients, 10 months -4 years of age, with premature thelarche (precocious breast tissue only). The latter two clinical groups did not have advanced physical or skeletal development.

Constitutional delay in adolescence and growth. The level of SLH was determined on 95 venous specimens obtained serially from 52 boys, $10-18 \% / 12$ years of age, with constitutional delay in growth and adolescence [24]. All had at least a 2-year delay in BA and a similar delay in HA plus a marked delay in onset of adolescence. Patients with possible or proven hypopituitarism have been excluded from this group. Most have been followed long enough to observe the ultimate onset of puberty in the untreated group and the maintenance of pubertal development after cessation of therapy (Depo-testosterone, $200 \mathrm{mg} \mathrm{im} / \mathrm{month}$ ) in the testosterone-treated group.

Adolescent gynecomastia. The level of SLH was determined on six males, 13-15 years of age, with gynecomastia and normal adolescent development. Since all had normal testicular development clinically and four of four tested were chromatin-negative, the diagnosis of Klinefelter's syndrome was excluded.

\section{Methods}

Values for SLH were determined by a modification of the double antibody radioimmunoassay method [13]. Details have beeen previously reported [8]. Sensitivity of the assay system, which uses logit transformation [17], permits accurate determination of values for $\mathrm{SLH}$ as low as $1.0 \mathrm{mIU} / \mathrm{ml}$ (Second International Reference Preparation of Human Menopausal Gonadotropin or 2nd IRP-HMG) when a 200- $\lambda$ sample size is employed. All values were determined in triplicate. Individual clinical groups had determinations of SLH levels performed in the same assay to avoid interassay variability within the groups. Interassay and intraassay variability ranged from $\pm 8 \%$ at a level of 45 $\mathrm{mIU} / \mathrm{ml}$ to $\pm 16 \%$ at a level of $1.5 \mathrm{mIU} / \mathrm{ml}$. 


\section{Results}

Gonadal Dysgenesis

The 131 SLH values obtained on 51 patients with gonadal dysgenesis are shown in figure 1. Differences in karyotype, not represented separately, did not influence the data. Similarly, Halotestin (fluoxymesterone) treatment has not been delineated separately, since no apparent effect on mean SLH values in the treated and untreated groups was observed with the dosages used $(2.5-5.0 \mathrm{mg} / 24 \mathrm{~h})$. The 14 values for 12 patients, 2-9 years of age, showed a mean SLH value of $4.5 \mathrm{~m} \mathrm{IU} / \mathrm{ml} \pm 1.15 \mathrm{SD}$, which was significantly higher $(\mathrm{p}<0.01)$ than the normal female mean for this age group $(2.7 \mathrm{mIU} / \mathrm{ml} \pm 0.6 \mathrm{SD})$. In the 10 - to 12 -year-old group, $75 \%$ of the SLH values were elevated, and the group mean was significantly higher $(p<0.01)$ than the normal mean for age. By age 13 , $92 \%$ of the values were significantly elevated $(p<0.01)$. Beyond this age, all the SLH values of patients not receiving estrogen therapy were greatly elevated above the normal level for age. Estrogen therapy apparently failed to suppress levels of SLH in $50 \%$ of the patients 16 years of age and older. This could not be readily

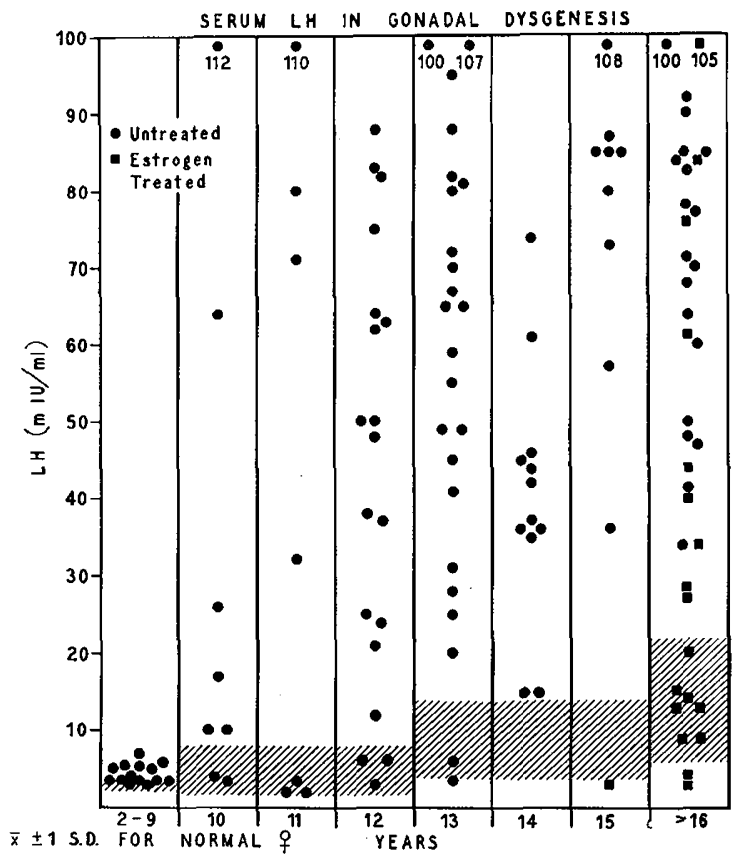

Fig.1. Serial measurement of SLH levels in 51 patients with gonadal agenesis compared with normal female mean $\pm 1 \mathrm{SD}$. Hatched areas indicate the range for normal values in this and subsequent figures. explained on the basis of duration of therapy, since many values were still elevated after many years of estrogen treatment. Most patients reportedly were receiving $1.25 \mathrm{mg}$ cyclic Premarin/24 h. Our data permitted neither further evaluation of the timing of venous sampling in relation to the days on which Premarin was taken, nor determination as to whether the estrogen was taken faithfully.

\section{Sexual Precocity, Premature Pubarche, and Premature} Thelarche

Figure 2 shows the values for SLH in females who are isosexually precocious. Thirteen values obtained from the sera of nine patients receiving Depo-provera (medroxyprogesterone), which was used in an attempt to delay sexual and physical maturation, are depicted separately. The presence or absence of menses at the time of venous sampling is also shown. Data rela ing to the presence or absence of ovulation in these patients were not available. Six of the nine receiving Depoprovera had previously menstruated, but all patients showed suppression of menses during therapy. For the untreated group, 2-9 years of age, the mean level of SLH of both menstruating and nonmenstruating patients was significantly higher $(p<0.01)$ than the normal mean for age. When the nonmenstruating patients were evaluated separately, this significant difference still persisted. In the Depo-provera-treated group, the mean level of SLH was also significantly higher $(p<0.01)$ than the normal mean for age. Unfortunately, accurate correlation of SLH values with the time of Depo-provera injection was not possible from our data. All patients, however, had received 200 mg sometime within the previous 14 days. The SLH values for all patients with idiopathic sexual precocity were in the normal range for stage of sexual development [8], irrespective of age.

Of the three patients with CNS disorders, two with hypothalamic dysfunction (resulting from head trauma and third ventricular tumor, respectively) had SLH values of 9.0 (mean of six determinations) and 8.0 $\mathrm{mIU} / \mathrm{ml}$ at ages of $7 \% /{ }_{12}$ and $910 / 12$ years, respectively. These values were elevated for age, but normal for stage of sexual development, since both patients were menstruating. A $9^{10} / 12^{-y e a r-o l d ~ p a t i e n t, ~ w h o ~ h a d ~ h a d ~}$ postmeasles encephalitis, showed a SLH value of 12.0 $\mathrm{mIU} / \mathrm{ml}$, which was elevated for both age and stage of sexual development, since she was not yet menstruating. The $8 \% 1 y^{1}$-year-old patient with McGuneAlbright's syndrome had irregular menses in the absence of any other sexual development for 8 months and a SLH value of $2.0 \mathrm{~m} \mathrm{IU} / \mathrm{ml}$ that was normal for age. The $97 / 12^{7}$-year-old patient with hypothyroidism had a SLH value of $2.5 \mathrm{mIU} / \mathrm{ml}$ just prior to the onset of menses; this value was also normal for age. 


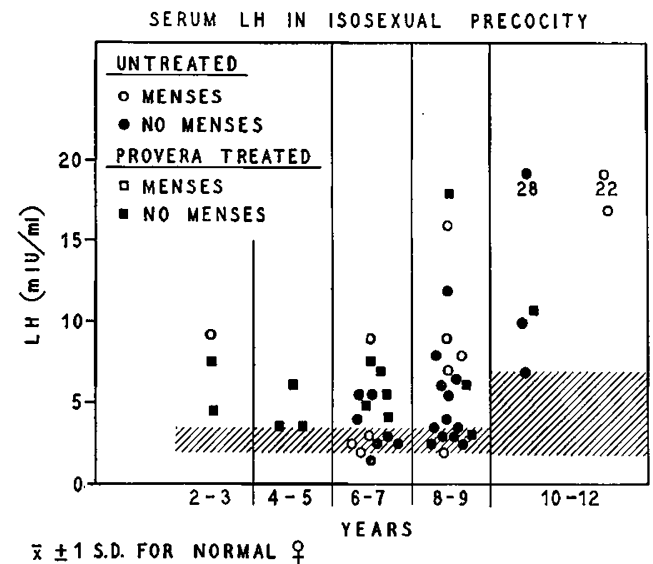

Fig. 2. SLH levels in 34 female patients with isosexual precocity.

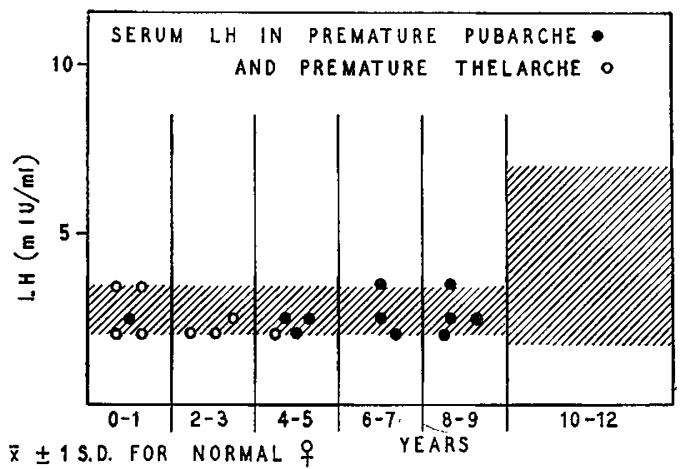

Fig.3. SLH levels in nine patients with premature pubarche and eight patients with premature thelarche.

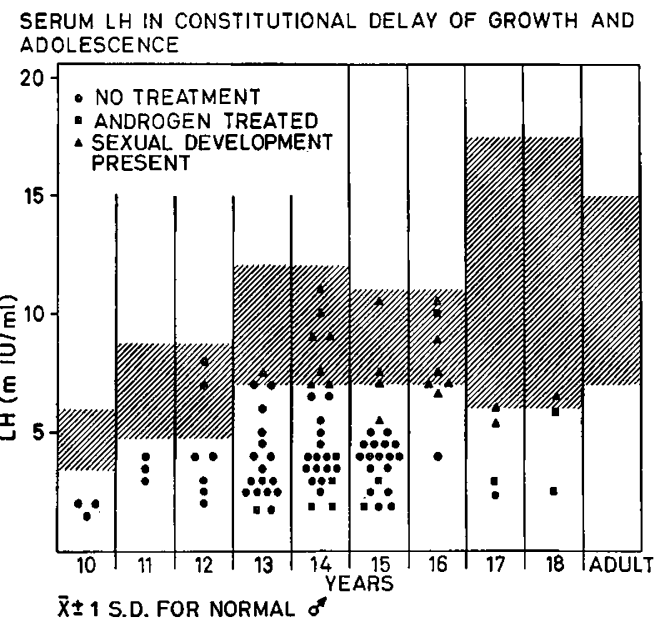

Fig.4. Serial measurement of SLH levels in 52 boys with constitutional delay in growth and adolescence compared with normal male mean $\pm 1 \mathrm{SD}$.
Figure 3 shows the results of 11 determinations of SLH values in nine patients with premature pubarche and in eight with premature thelarche. All values were in the normal range for age.

\section{Constitutional Delay in Growth and Adolescence}

Figure 4 shows the 95 SLH values obtained from 52 boys with adolescent delay in growth and development. The patients, aged $11-12,13-14$, and 15-16 years, all had group means for SLH levels that were significantly below $(p<0.01)$ the normal mean for age. Once significant sexual development appeared [23], all values were in the normal range for age. In this group of patients, this did not occur until 14 years of age or later.

Exogenous testosterone therapy was associated with lowered SLH levels in all patients except for one 16year-old. This may be a result of therapy or, alternatively, the patients may have had endogenously low values similar to those in the untreated group.

\section{Adolescent Gynecomastia}

SLH values obtained from six patients, $13-15$ years of age, with adolescent gynecomastia were in our normal adult range of $4.0-25.0 \mathrm{mIU} / \mathrm{ml}$ and were not those expected in Klinefelter's syndrome [18, 19].

\section{Discussion}

Serum gonadotropin determinations obtained using radioimmunoassay techniques must be interpreted with caution for several reasons. These determinations cannot necessarily be expected to correlate with those obtained using biological techniques, as different sites of the molecule probably cause different actions [1]. No serum standard for LH values is currently available, and the urinary or pituitary standards routinely used could have characteristics that differ from those of circulating gonadotropins. In addition, considerable variation in values may arise because of the use of different antisera [3], because of individual patient variation within the same $24-\mathrm{h}$ period $[15,19]$, and because of interlaboratory variation even with the use of the same materials [2]. If, however, each laboratory establishes its own quality controls [17], groups of subjects with normal gonadotropin metabolism can be compared with those having various disorders of sexual development.

Using such quality controls and realizing the limitations of the methodology, we have reported previously [8] that normal male and female children have measurable LH levels in their sera by 3 years of age and that the mean value for each group increases at age 10-11 and progressively increases until age 13-14, when both 
boys and girls obtain normal adult values. Except for the ovulatory peak in midcycle, SLH values in males and females are comparable. The normal mean SLH values $\pm 1 \mathrm{SD}$ for various ages are given in figures 1 and 4 .

We also have reported a progressive increase in SLH values with the various stages of sexual development [8]. The mean SLH values for groups of girls classified according to stage of sexual development reach adult non midpeak levels $(3.0-22 \mathrm{mIU} / \mathrm{ml})$ at the time of menarche. Adult levels are reached in males by the time they reach stage four of the five stages of sexual development [23]. By comparing these data with those from groups of individuals with abnormal sexual development, certain conclusions can be reached.

Girls with gonadal dysgenesis, as a group, have slightly elevated SLH values during childhood, but determinations of SLH values cannot be used for diagnostic purposes before the age of 9-13 years. Silver [22] and Grossman [5], using bioassay techniques, previously demonstrated increased urinary gonadotropin excretion for age in a few such patients. Similar findings by radioimmunoassay have been reported by others $[14,18]$. Once elevation occurred in our patients, the values quickly rose to postmenopausal levels. Fifty percent of the older patients, reportedly receiving cyclic estrogen therapy, had SLH values above normal adult levels. Difficulty in suppressing the elevated LH levels of postmenopausal women has been reported [20]. Perhaps larger doses of estrogen or the addition of other ovarian steroids, such as progesterone, are needed to produce more complete suppression of $\mathbf{L H}$.

Constitutional (idiopathic) sexual precocity presumably results from the early activation of the hypothalamic-pituitary-gonadal axis which is responsible for normal pubescence. Measurement of urinary gonadotropins by bioassay in such patients has revealed elevated levels in some cases and nondetectable levels in others [4, 6, 9, 21, 24]. Root et al. [18], using radioimmunoassay techniques, reported low or undetectable LH levels in sera of patients with sexual precocity, premature pubarche, and premature thelarche. In contrast, KeNNY et al. [10] reported increased SLH values in 5 patients (4 females and 1 male) with idiopathic sexual precocity and significantly increased SLH values in 15 patients with premature thelarche. Our data reveal considerable overlap of SLH values in patients with sexual precocity; these values are in the normal range for age. In the presence of menses, the SLH value was more consistently, but not necessarily, elevated for age. Thus, the finding of a normal SLH value for age did not preclude the diagnosis of idiopathic sexual precocity. In several cases, subsequent serial determinations have revealed values that were elevated for age. The three patients with hypothalamic dysfunction all showed increased values for age, whereas the patient with McCuneAlbright's syndrome and the patient with hypothyroidism plus sexual precocity had normal values for age. In all our patients with premature pubarche and premature thelarche, SLH values were normal for age. Selection of patients with sexual precocity without regard to age, etiology, or menstrual history may have led to the variable results reported.

Progesterone and medroxyprogesterone reportedly have suppressed pituitary gonadotropins, as determined by bioassay estimates of pooled urine extracts $[9,11,12,25]$. Incomplete suppression of SLH values to normal levels for age was observed in many of our patients receiving $200 \mathrm{mg}$ of medroxyprogesterone by intramuscular injection every 2 weeks. KENNY et al. [10] reported similar results in one patient. Unfortunately, it was impossible to document from our records the time relation of the last injection to the time the serum was obtained. All patients, however, had received an injection within the previous 14 days and did not menstruate; most had clinical regression of estrogenic effects. The injection had less effect on physical and skeletal maturation.

Height and bone age were retarded for all the patients with constitutional delay in growth and adolescence. A few older patients showed early sexual development despite the retardation. Nearly all those with sexual infantilism had values greater than $1 \mathrm{SD}$ below the mean for age (fig.4). Those with significant sexual development uniformly had values within $1 \mathrm{SD}$ of the mean for age. Those treated with androgens showed low values. These data agree with those reported by Root et al. [18].

Mechanisms of normal puberty continue to be mostly speculative. The small, but statistically significant, elevation of SLH levels in patients with gonadal agenesis may suggest that the ovary plays an important feedback role at an early age. Hypothalamic-releasing factors, however, obviously are not under significant ovarian control until approximately $9-12$ years of age. Sexual development appears to usually parallel the level of serum gonadotropins. Further studies are necessary to elucidate the controlling factors for gonadotropin release at adolescence.

\section{Summary}

Using a double antibody radioimmunoassay method, SLH values were determined in children with various disorders of adolescent sexual development. Patients with gonadal dysgenesis had a significantly increased mean SLH value at all ages, including the prepubertal ages of 2-9 years. Patients with idiopathic female 
sexual precocity had mean SLH values that were elevated for age, but consistent with their stage of sexual development. Medroxyprogesterone therapy apparently did not suppress SLH levels. In patients with constitutional delay of adolescence and growth, SLH values were uniformly below normal for age, but again consistent with stage of sexual development. Patients with premature thelarche, premature pubarche, and adolescent gynecomastia showed normal SLH values for age. The determination by radioimmunoassay of the level of serum gonadotropins in patients with disorders of adolescent sexual development can aid in diagnosis. SLH levels and stage of sexual development correlate well.

\section{References and Notes}

1. Albert, A.: Bioassay and radioimmunoassay of human gonadotropins. J. clin. Endocrin. 28: 1683 (1968).

2. Albert, A.; Rosemberg, E. ; Ross, G.T.; Paulsen, C.A. and RyAN, R.J.: Report of the National Pituitary Agency collaborative study on the radioimmunoassay of FSH and LH. J.clin. Endocrin. 28: 1214 (1968).

3. Gargille, C.M.; Rodbard, D. and Ross, G.T.: Radioimmunoassay of human follicle stimulating hormone: Bias due to antisera. J.clin.Endocrin. 28: 1276 (1968).

4. Donovan, B.T. and van DeR Werff ten Bosch, J.J.: The physiology of puberty (Williams and Wilkins, Baltimore 1965).

5. Grossman, E. R.: Pituitary gonadotropins in gonadal dysgenesis. Age of appearance and variability in amount of follicle stimulating hormone (FSH) in urine. Pediatrics 25: 298 (1960).

6. HaIn, A. M.: The constitutional type of precocious puberty. J.clin. Endocrin. 7: 171 (1947).

7. Johanson, A.J.;BRasel, J. A. and Blizzard, R. M. : Growth in patients with gonadal dysgenesis receiving fluoxymesterone. J. Pediat. (1969) (in press).

8. Johanson, A. J. ; GuYda, H. J. ; Light, C. ; Mrgeon, G.J. and Blizzard, R.M.: Serum luteinizing hormone $(\mathrm{LH})$ by radioimmunoassay in normal children. J. Pediat. 74: 416 (1969).

9. Kaplan, S. A.; Ling, S. M. and IranI, N. G.: Idiopathic isosexual precocity. Therapy with medroxyprogesterone. Amer. J. Dis. Child. 116: 591 (1968).

10. Kenny, F.M.; Midgley, A.R.; Jaffe, R.B.; Garces, L.Y. and VAsQuez, A.: Radioimmunoassay of luteinizing hormone $(\mathrm{LH})$ and follicle stimulating hormone (FSH) in normal children and various abnormal conditions (Abstract). J. Pediat. 72: 565 (1968).
11. Kulin, H.E.; Rrfkind, A.B. and Ross, G.T.: Human luteinizing hormone (LH) activity in processed and unprocessed urine measured by radioimmunoassay and bioassay. J.clin. Endocrin. 28: 543 (1968).

12. Kupperman, H.S. and Epstein, J.A.: Medroxyprogesterone acetate in the treatment of constitutional sexual precocity. J.clin. Endocrin. 22: 456 (1962).

13. Mrdgley, A.R.: Radioimmunoassay: A method for human chorionic gonadotropin and human luteinizing hormone. Endocrinology 79: 10 (1966).

14. Odell, W.D.; Ross, G.T. and Rayford, P.L.: Radioimmunoassay for luteinizing hormone in human plasma or serum: Physiologic studies. J. clin. Invest. 46: 248 (1967).

15. Peterson, N.T., Jr.; Mrdgley, A.R., Jr. and JAFFE, R.B.: Regulation of human gonadotropins. III. Luteinizing hormone and follicle stimulating hormone in sera from adult males. J. clin. Endocrin. 28: 1473 (1968).

16. Raiti, S.; Johanson, A.J.; Light, C.; Migeon, C.J. and Blizzard, R.M.: Follicle stimulating hormone in serum of males from childhood through maturity to adulthood, as measured by radioimmunoassay. Metabolism (1969) (In press).

17. Rodbard, D.; RAYFord, P.L.; CoOper, J.A. and Ross, G.T.: Statistical quality control of radioimmunoassays. J.clin. Endocrin. 28: 1412 (1968).

18. Root, A.W.; Moshang, T., Jr.; Bongrovanni, A.M. and EBerLeIN, W.R.: The relationship of plasma luteinizing hormone $(\mathrm{LH})$ concentration to gonadal function (Abstract). 78th meet. Amer. Pediat. Soc., Atlantic City, N.J., 1968, Progr., p. 21, 1968.

19. Saxena, B.B.; Demura, H.; Gandy, H.M. and Peterson, R.E.: Radioimmunoassay of human follicle stimulating and luteinizing hormones in plasma. J.clin. Endocrin. 28: 519 (1968).

20. Schalch, D.A.; Parlow, A.F.; Boon, R. C. and ReIchlin, S.: Measurement of human luteinizing hormone in plasma by radioimmunoassay. J. clin. Invest. 47: 665 (1968).

21. Sigurjonsdottir, T.J. and Hayles, A.B.: Precocious puberty. A report of 96 cases. Amer. J. Dis. Child. 115: 309 (1968).

22. Srlver, H.K.: Syndrome of ovarian agenesis (congenitally aplastic ovaries). Pediatrics $8: 368$ (1951).

23. TANNER, J. M.: Growth at adolescence. (Blackwell and Mott, Oxford 1962).

24. WrLkrns, L.: The diagnosis and treatment of endocrine disorders in childhood and adolescence; 3rd ed. (Thomas, Springfield, Ill. 1965).

25. Williams, R.H.: Textbook of endocrinology; 4th ed. (Saunders, Philadelphia 1968). 
26. We wish to express our appreciation to Dr. LEo REICHERT for the immunochemical hormone and to Dr. Rees Midgley for the HCG antiserum. The 2nd IPR-HMG was supplied by Dr. D. BANGHAM, National Institute for Medical Research, Mill Hill, London. The National Pituitary Agency, the Endocrinology Study Section, and the National Institutes of Arthritis and Metabolic Diseases made these studies possible. Mrs. Mary Westervelt provided valuable secretarial assistance.
27. Supported by Research Grants nos. HD 01852 , AM 00180-16 and M6-327003, Traineeship Grant no. TI-AM-5219 (AJJ), the Medical Research Council of Canada (HJG) and Career Research Award no. JK 06 AM 21, 855 (GJM) of the United States Public Health Service.

28. Requests for reprints should be addressed to RoBERT M. Blizzard, M.D., The Ghildren's Medical and Surgical Center, The Johns Hopkins Hospital, Baltimore, Maryland 21205 (USA). 